\title{
Improvement in Glucose Regulation Using a Digital Tracker and Continuous Glucose Monitoring in Healthy Adults and Those with Type 2 Diabetes
}

\author{
Ashkan Dehghani Zahedani · Solmaz Shariat Torbaghan · \\ Salar Rahili · Kirill Karlin · Darrin Scilley · Riya Thakkar • \\ Maziyar Saberi · Noosheen Hashemi · Dalia Perelman • \\ Nima Aghaeepour • Tracey McLaughlin • Michael P. Snyder
}

Received: March 30, 2021 / Accepted: May 12, 2021 / Published online: May 28, 2021

(C) The Author(s) 2021

\section{ABSTRACT}

Introduction: While continuous glucose monitoring (CGM) has been shown to decrease both hyper- and hypoglycemia in insulin-treated diabetes, its value in non-insulin-treated type 2 diabetes (T2D) and prediabetes is unclear. Studies examining the reduction in hyperglycemia with the use of CGM in non-insulintreated T2D are limited.

Methods: We investigated the potential benefit of CGM combined with a mobile app that links each individual's glucose tracing to meal composition, heart rate, and physical activity in a cohort of 1022 individuals, ranging from nondiabetic to non-insulin-treated T2D, spanning a wide range of demographic, geographic, and socioeconomic characteristics. The primary endpoint was the change in time in range (TIR), defined as $54-140 \mathrm{mg} / \mathrm{dL}$ for healthy and

Ashkan Dehghani Zahedani and Solmaz Shariat Torbaghan contributed equally.

Supplementary Information The online version contains supplementary material available at https:// doi.org/10.1007/s13300-021-01081-3.

A. Dehghani Zahedani $\cdot$ S. Shariat Torbaghan .

S. Rahili · K. Karlin · D. Scilley · R. Thakkar .

M. Saberi · N. Hashemi · D. Perelman ·

N. Aghaeepour · T. McLaughlin · M. P. Snyder ( $\varangle)$ January AI, 1259 El Camino Real \#231, Menlo Park, CA 94025, USA

e-mail: mike@mail.january.ai prediabetes, and $54-180 \mathrm{mg} / \mathrm{dL}$ for T2D, from the beginning to end of a 10-day period of use of the Freestyle Libre CGM. Logged food intake, physical activity, continuous glucose, and heart rate data were captured by a smartphone-based app that continuously provided feedback to participants, overlaying daily glucose patterns with activity and food intake, including macronutrient breakdown, glycemic load (GL), and glycemic index (GI).

Results: A total of 665 participants meeting eligibility and data requirements were included in the final analysis. Among self-reported nondiabetic participants, CGM identified glucose excursions in the diabetic range among $15 \%$ of healthy and $36 \%$ of those with prediabetes. In the group as a whole, TIR improved significantly $(p<0.001)$. Among the $51.4 \%$ of participants who improved, TIR increased by an average of $6.4 \%(p<0.001)$. Of those with poor baseline TIR, defined as TIR below comparable A1c thresholds for T2D and prediabetes, 58.3\% of T2D and $91.7 \%$ of healthy/prediabetes participants improved their TIR by an average of $22.7 \%$ and $23.2 \%$, respectively. Predictors of improved response included no prior diagnosis of T2D and lower BMI.

Conclusions: These results indicate that 10-day use of CGM as a part of multimodal data collection, with synthesis and feedback to participants provided by a mobile health app, can significantly reduce hyperglycemia in non-insulin-treated individuals, including those with 
early stages of glucose dysregulation.Keywords: Continuous glucose monitor; Diabetes; Digital health; Prediabetes; Time in range

\section{Key Summary Points}

The clinical benefit of continuous glucose monitoring (CGM) in non-insulin-treated type 2 diabetes (T2D) and those with earlier stages of glucose dysregulation is controversial and relatively unstudied.

In the largest study to date on the above population, we showed that in 665 individuals with non-insulin-treated T2D, prediabetes, or no history of diabetes, 10-day use of the Freestyle Libre CGM and the Sugar AI mobile app that integrates CGM data with food logging/physical activity and provides continuous feedback to users, TIR improved significantly in both T2D and those without diabetes. Among those with low baseline TIR, improvement was $22.7 \%$ and $23.2 \%$, respectively, for $\mathrm{T} 2 \mathrm{D}$ and nondiabetic individuals.

Individuals without a prior diagnosis of T2D and lower body mass index (BMI) showed the greatest response to the intervention.

Results from this study indicate that shortterm use of CGM along with exposure to activity and food insights can significantly improve TIR in participants with noninsulin-treated T2D and earlier stages of glucose dysregulation.

\section{DIGITAL FEATURES}

This article is published with digital features, including a summary slide, to facilitate understanding of the article. To view digital features for this article go to https://doi.org/10.6084/ m9.figshare.14561931.

\section{INTRODUCTION}

Type 2 diabetes (T2D) is a growing problem worldwide and the prevalence continues to increase. Presently, 34.2 million US adults have T2D and another 88 million have prediabetes, the majority of whom are unaware of their diagnosis [1]. Of those with prediabetes, 70\% will convert to T2D during their lifetime [2]. As demonstrated in the Diabetes Prevention Program (DPP), lifestyle modifications, including $7 \%$ loss of initial body weight and $150 \mathrm{~min}$ of exercise per week, are highly effective in preventing transition to T2D in high-risk individuals [3] and lower hemoglobin A1c in individuals with T2D [4]. The success of this intervention, however, is labor-intensive, requiring individual case managers, frequent contact with participants, a 16-session core curriculum, supervised physical activity session, and extensive network of training, feedback, and support [5]. These labor-intensive requirements limit the reach and efficacy of programs. Indeed, a meta-analysis of 28 National DPP translational interventions showed an average weight loss of only $4 \%$ with attrition rates as high as 50\% [6]. Technology-enabled diabetes self-management approaches have gained traction as supplements or alternatives to traditional diabetes self-management models, with demonstrated improvement in HbA1c [7]. These technologies provide a platform for the development of personalized, patient-centered interventions that integrate patient-generated health data, tailored education, and individualized feedback, which are effective behavior interventions that extend access and feasibility $[8,9]$. Thus, new strategies that harness the benefits of technology aimed at preventing and/ or treating $\mathrm{T} 2 \mathrm{D}$ have the potential to address gaps in current approaches to lifestyle interventions and to do so at scale.

Continuing glucose monitoring (CGM) has demonstrated efficacy in improving HbA1c and reducing hypoglycemia in patients with type 1 diabetes and insulin-treated T2D [10-17]. Flash glucose monitoring (FGM) enables patients to gain immediate information about current glucose and trend (upward or downward) as well as 
a continuous curve showing glucose pattern after swiping a Bluetooth receiver across an implanted sensor. Real-time CGM (rtCGM) does not require a swipe, and has the added benefit of alarms set to alert patients to highs and lows. Immediate feedback on glucose patterns from both forms of CGM conveys a wealth of information to the user about the impact of specific foods, exercise, sleep, stress, and combinations thereof, with potential for expanded use in noninsulin-treated T2D and prediabetes as a tool to support lifestyle changes aimed at reducing hyperglycemia $[13,18]$. Few studies have addressed this. Three published studies have demonstrated a benefit of rtCGM in non-insulin-treated $\mathrm{T} 2 \mathrm{D}$ in facilitating behavior change, weight reduction, increased physical activity, decreased calorie, carbohydrate, and/or high glycemic index food consumption, increased fiber intake, and significant reductions in HbA1c [19-21], and only one published study in non-insulin-treated T2D showed benefit from flash CGM in reducing HbA1c [22]. Thus, more research is warranted to explore the utility of CGM in facilitating healthy lifestyle changes in early-stage diabetes/prediabetes. The Sugar Challenge Study was designed to examine whether a 10-day use of CGM together with a mobile app (Sugar AI), which captures dietary and physical activity data, integrates these with glucose patterns, and provides continuous feedback, improves hyperglycemia.

We performed an observational study in a cohort of 1022 individuals, spanning a wide range of demographic, geographic, socioeconomic backgrounds, and ranging from normoglycemic to non-insulin-treated T2D, in which CGM was combined with a mobile app (Sugar AI) to provide feedback on each individual's glucose patterns, along with food composition, heart rate, and physical activity. Time in range (TIR), a relatively new and validated measure of hyperglycemia [14, 23], was used to quantify short-term benefits in daylong glycemia, and clinical factors that predicted improvement in TIR were identified. We found a significant improvement in TIR not only in individuals with T2D but also in healthy individuals with earlier stages of glucose dysregulation (prediabetes and normoglycemia who on CGM exhibited postprandial excursions outside the normal range). This is the first study to demonstrate a significant reduction in hyperglycemia as a result of CGM use, and the largest to demonstrate a significant reduction in hyperglycemia in non-insulin-treated T2D. It is the first to evaluate the integration of CGM with data gathered by a mobile app that tracks lifestyle factors such as food and physical activity, and provides users with continuous integrated data that may enhance behavior modification.

\section{METHODS}

\section{Study Overview}

The Sugar Challenge Study was designed to ascertain whether the use of the Sugar AI app along with CGM could reduce hyperglycemia in individuals with early stages of glucose dysregulation who might benefit from lifestyle changes. The Sugar AI app allows participants to log their food, water, medication, and physical activity. Glycemic index (GI), load (GL), and macronutrients for each logged food were calculated and presented information back to the participants in addition to daily summaries of nutrition and activity patterns in relation to glycemic excursions. The Sugar AI app did not provide specific dietary or food recommendations. This prospective, unblinded observational trial included volunteers from 47 US states plus the District of Columbia, spanned a wide demographic range, and included a wide range of glycemia, from healthy individuals to those with prediabetes or non-insulin-treated T2D (Table 1). Participants were provided a heart rate monitoring (HRM) device and Abbott Libre CGM and were instructed to wear them for 10 consecutive days while scanning their sensor with Abbott's LibreLink app at least once every $8 \mathrm{~h}$ to maintain signal capture. They were also asked to log their physical activity, food, medication, and water consumption. Participants were subsequently able to visualize glucose curves, along with nutritional and activity summaries (Fig. 1), on the Sugar AI app on their mobile device. The Sugar AI app was designed 
Table 1 Breakdown of subjects according to age, sex, race, geographic location, BMI, and fasting glucose in the total cohort and subgroups defined as healthy, prediabetes, and type 2 diabetes based on their self-claimed classification

\begin{tabular}{lllll}
\hline Characteristic & $\begin{array}{l}\text { Healthy } \\
(\boldsymbol{n}=\mathbf{4 4 8})\end{array}$ & $\begin{array}{l}\text { Prediabetes } \\
(\boldsymbol{n}=\mathbf{2 5})\end{array}$ & $\begin{array}{l}\text { Type 2 diabetes } \\
(\boldsymbol{n}=\mathbf{1 9 2})\end{array}$ & $\begin{array}{l}\text { Entire group } \\
(\boldsymbol{n}=\mathbf{6 6 5})\end{array}$ \\
\hline Geographic region & 51 & 3 & 25 & 79 \\
Northeast & 97 & 5 & 89 & 191 \\
South Midwest & 83 & 4 & 48 & 135 \\
West & 93 & 12 & 29 & 134 \\
Age (years) & $34.6 \pm 9$ & $35.8 \pm 7.7$ & $43.1 \pm 8.8$ & $37.7 \pm 9.7$ \\
Sex (F/M) & $202 / 125$ & $15 / 10$ & $137 / 55$ & $354 / 190$ \\
Race & 215 & 11 & 139 & 365 \\
White/Caucasian & 27 & 5 & 28 & 60 \\
Black American & 30 & 5 & 19 & 54 \\
Asian American & 38 & 2 & 2 & 42 \\
Indian or Alaska Native & 2 & 0 & 1 & 3 \\
Native Hawaiian/Other Pacific & 2 & 1 & 0 & 3 \\
$\quad$ Islander & & & 3 & $32.5 \pm 8.9$ \\
Other & 13 & 1 & $39.2 \pm 8.5$ & $87.8 \pm 33.6$ \\
BMI (kg/m ${ }^{2}$ ) & $28.5 \pm 6.7$ & $34.5 \pm 5.4$ & $115.1 \pm 47.3$ & \\
Fasting glucose & $76.6 \pm 16.1$ & $79.9 \pm 14.9$ & &
\end{tabular}

by January AI specifically for this study. The primary endpoint of the study was a comparison of glucose TIR after 10 days of use as compared with baseline.

\section{Participants}

Recruitment for the study occurred via social media channels and online classified ads, targeting individuals from diverse geographic, socioeconomic, age, and education levels. Respondents were required to provide written informed consent and complete an online screening demographic and health questionnaire. Eligible participants could be healthy or have a diagnosis of prediabetes or non-insulindependent T2D (Table 1). Eligible participants were required to wear a HRM device 20-24 h/day, and flash CGM for 10 consecutive days, provide body weight at baseline, and agree to comprehensive logging of food intake, physical activity, medication, and water consumption for 10 consecutive days using the Sugar AI app. Individuals who met the online eligibility criteria were further screened by clinical coordinators via telephone who confirmed eligibility and completed enrollment. Individuals were excluded from the study if they did not meet the criteria described above or if any of the following criteria were met: use of vitamin C supplements in excess of $200 \%$ of the US recommended daily allowance at least 14 days prior to starting the trial; allergy to skin adhesives used in the trial; women who were pregnant, lactating, had given birth in the past 6 months, or were planning to get pregnant in the next 6 months; and individuals who were taking any of the following medications: insulin, oral hypoglycemic medications (sulfonylureas, meglitinides), progesterone, atypical 
a

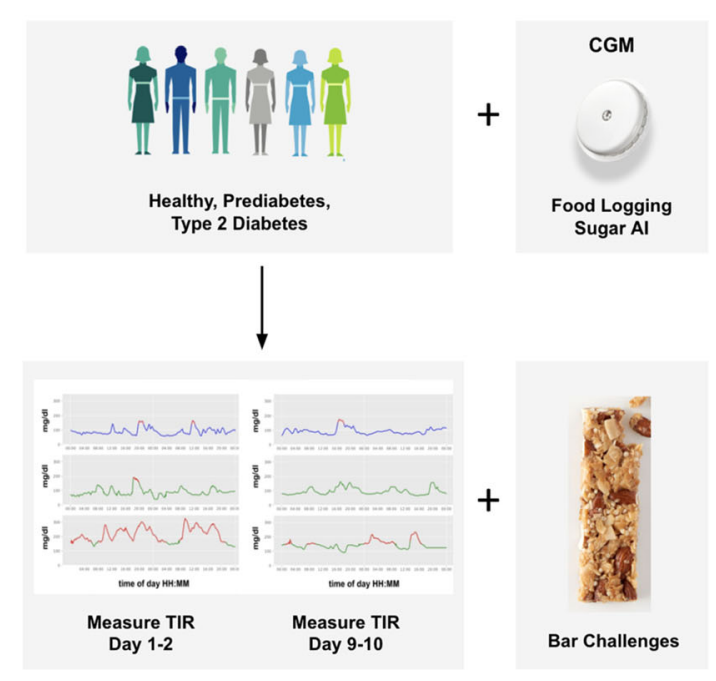

Fig. 1 Study design, Sugar AI app, and subject selection. a Ten-day study design to evaluate improvement in time in range (TIR) as a result of continuous glucose monitoring (CGM) and a mobile app (Sugar AI) that integrates glucose curves with eating and physical activity patterns in healthy nondiabetics and those with prediabetes or non-

antipsychotics, oral corticosteroids, triphasic oral contraceptives, blood thinners, deemed unfit for participation by the study coordinator, and allergy to nuts. Of the 192 people with diabetes 152 took medication; of these, 97 were on metformin and the rest were on other medications. Participants that completed the Sugar Challenge Study as outlined were remunerated with a US \$50 Amazon gift card.

Because not all subjects who completed the 10-day study wore the CGM continuously as instructed, for the purposes of analysis, it was predetermined that only those who wore the CGM for at least 9 days with at least 33\% daily coverage were included in the final analysis. In accordance with these criteria, 665 subjects out of 1022 enrolled were included in this study.

\section{Intervention}

Enrolled participants were mailed an Abbott Freestyle Libre flash CGM (henceforth referred to as (GM) a MiBand 3 or Garmin watch to record heart rate (HRM), and instructed to

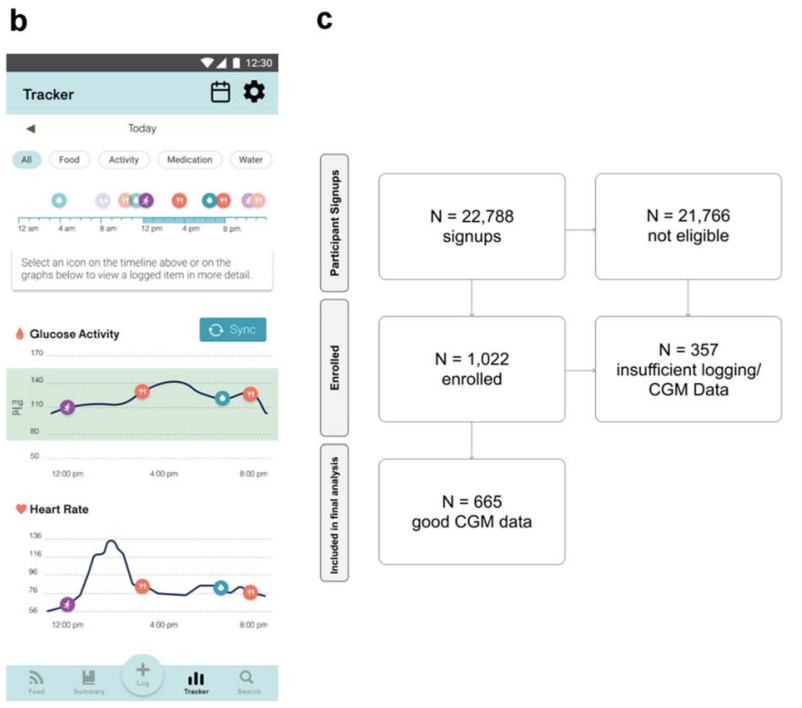

insulin-treated type 2 diabetes. b Snapshot of the Sugar AI app designed for this trial. Users visualize their concurrent food and activity logs on top of their CGM and heart rate signals. c Diagram showing the number of participants recruited and the number of participants that were included in this study

download the Sugar AI app through which they could search a large food database of over 15 million foods and log all food, physical activity, water, and medications taken. Participants were provided with two nutritional bars and a dextrose solution (TRUEplus, or CVS Health Glucose Shot; $15 \mathrm{~g}$ ) to generate glycemic responses to standardized nutrient challenges. The Sugar AI food database contains nutritional information for each logged food. GI and GL values calculated through January AI technology were also available for each food in the database. Participants were guided to scan their CGM using the Abbott LibreLink app which retrieves up to $8 \mathrm{~h}$ of CGM values. Aside from the standard glucose drink (day 3) and the two nutritional bars, participants determined their own dietary and activity patterns but were required to log their food, water, and physical activity for the duration of the study. No dietary recommendations were made while enrolled in the Sugar Challenge Study.

GI, GL, total calories, macronutrient content, along with heart rate, and water consumption were displayed to the participants via 
a tracker feature that overlays multiple data components on the CGM measurements and heart rate signals (Fig. 1b). The overlay of nutritional components of logged foods on CGM glucose curves was presented to the subjects at the time of logging. To encourage participant engagement, a point system was devised, in which participants were encouraged to attain at least 10,000 points by logging food, water, medication, and physical activity (Supplementary Table S1).

\section{Continuous Glucose Monitoring}

The Abbott Freestyle Libre was provided at no cost to participants who were instructed to apply a 10-day sensor to the back of the arm following the manufacturer's written instructions and demonstration video. Clinical coordinators were available by phone, video conferencing, or in-person to troubleshoot CGM sensor placement for those who had difficulties. The small CGM subcutaneous sensor measures interstitial glucose values every 15 min which can be transmitted using nearfield communications. Participants obtained their glucose values after scanning for up to $8 \mathrm{~h}$ of data (i.e., Flash CGM). No alarms were used.

\section{Structured Food Challenges}

During the 10 days of monitoring, all subjects were asked to consume three standardized nutrient challenges in order to identify patterns of glycemia that were not dependent on variability in nutrients consumed:

1. Oral glucose challenge: on day 3 while wearing the CGM, after fasting for at least $10 \mathrm{~h}$, participants $(n=328)$ drank over $5 \mathrm{~min}$ three $15-\mathrm{g}$ portions of dextrose solution (TRUEplus or CVS Health Glucose Shot), followed by $2 \mathrm{~h}$ of inactivity to obtain a standardized glycemic response pattern via CGM.

2. Mixed meal challenges: on day 5 of the challenge after fasting for at least $10 \mathrm{~h}$, participants $(n=212)$ consumed a highprotein bar (Garden of Life; S'mores) with the following nutritional content: $200 \mathrm{cal}$ per serving [total carbohydrates (25 g), fiber $(14 \mathrm{~g})$, protein $(14 \mathrm{~g})$, and total fat $(8 \mathrm{~g})]$ followed by $2 \mathrm{~h}$ of inactivity to obtain standardized glycemic response pattern via CGM. On day 7 of the challenge after fasting for at least $10 \mathrm{~h}$, participants $(n=286)$ consumed a Kind bar (Dark Chocolate Nuts \& Sea Salt) with the following nutritional content: $180 \mathrm{cal}$ per serving [total carbohydrates $(16 \mathrm{~g})$, fiber $(7 \mathrm{~g})$, protein $(6 \mathrm{~g})$, and total fat $(15 \mathrm{~g})]$.

\section{Assessment of Time in Range}

As a result of potential inaccuracy of CGM during the first and last $24 \mathrm{~h}$ of sensor use, we defined the change in percentage TIR during days 8-9 relative to the percentage TIR at baseline which was defined as days 2-3 (excluding day 3 for those who took the glucose shot on day 3) of the 10-day study period. TIR was defined as the percentage of time glucose measurements were $54-140 \mathrm{mg} / \mathrm{dL}$ (3.0-$7.8 \mathrm{mmol} / \mathrm{L}$ ) for healthy and prediabetes and $54-180 \mathrm{mg} / \mathrm{dL}(3.0-10.0 \mathrm{mmol} / \mathrm{L})$ for diabetes. We used $54 \mathrm{mg} / \mathrm{dL}$ as the lower limit for TIR as healthy individuals can have nonpathologic glucose values below $70 \mathrm{mg} / \mathrm{dL}$. The American Diabetes Association has defined $54 \mathrm{mg} / \mathrm{dL}$ [24] as clinically significant hypoglycemia and this limit is used to diagnose hypoglycemic disorders in patients who are not taking glucoselowering medications [24]. Thus, we chose this lower limit so as to prevent overclassification of hypoglycemia in nondiabetic individuals. Upper limits of $140 \mathrm{mg} / \mathrm{dL}$ and $180 \mathrm{mg} / \mathrm{dL}$ were chosen for healthy/prediabetes and T2D, respectively.

\section{Defining the Group with Poor TIR at Baseline}

Because our analysis was to determine whether the use of CGM could improve TIR, we conducted a secondary analysis on individuals with suboptimal glucose patterns defined as follows: for healthy and those with prediabetes we used TIR of less than $83 \%$, which corresponds to A1c 
of less than $5.7 \%$, and for those with T2D we used TIR of less than $72 \%$, which corresponds to A1c of less than $6.5 \%[25,26]$.

\section{Responders and Non-Responders}

Self-reported demographic and clinical characteristics of the best and worst responders were compared to determine which participant might be the best candidate for use of CGM plus Sugar AI app. Responders were defined as the top quartile of change in TIR (from baseline to end of study), and non-responders were defined as the bottom quartile of change in TIR within the subgroups with suboptimal baseline glucose profiles as defined above.

\section{Statistical Analysis}

The TIR for the last 2 days vs TIR for the first 2 days of complete data capture was analyzed via the nonparametric Wilcoxon signed-rank test [27, 28]. Mann-Whitney rank test [29] was used to compare the continuous variables (e.g., age, BMI) between the good responders and bad responders. Comparisons were considered statistically significant with $p<0.05$. To account for the statistical analyses of multiple independent variables in this study, the Benjamini-Hochberg procedure $(\mathrm{BH})$ was employed to control the false discovery rate (FDR) $[30,31]$ at the level of 0.05 . These analyses were performed on the subset of participants who provided the required information detailed in Table 1.

\section{Compliance with Ethics Guidelines}

All named authors meet the International Committee of Medical Journal Editors (ICMJE) criteria for authorship for this article, take responsibility for the integrity of the work as a whole, and have given their approval for this version to be published. This study was performed in accordance with the Helsinki Declaration of 1964 and its later amendments. The study was approved by WCG New England IRB 120190429.

\section{RESULTS}

\section{The Sugar Challenge Study Cohort}

A total of 22,788 subjects responded to public advertisements and were assessed for eligibility (Fig. 1c). Of these, 1022 individuals who selfreported as healthy (without diabetes) or were diagnosed with prediabetes or non-insulin-dependent T2D were enrolled in the study. The final study cohort included 665 participants who met the pre-designated data quality requirements, including nine or more days of complete CGM data (Fig. 1c; see "Methods"). Of these 665 subjects, 448 were self-reported as healthy (without diabetes), 25 with prediabetes, and 192 with T2D (Table 1). Our diverse subject pool included women $(n=354)$, men $(n=190)$, and 121 with unreported sex, with a median age of 36 years old, and a median BMI of $30.9 \mathrm{~kg} /$ $\mathrm{m}^{2}$. Geographic, ethnicity, and other information are summarized in Table 1 . On average, participants logged 10.7 food and activity entries per day, 50\% more than required by the study criteria. In total, 25 million data points were captured across participant logs, heart rate, and continuously measured glucose data.

\section{Many Healthy Participants Exhibit Glucose Dysregulation}

We first examined the fasting glucose levels of the participants using the CGM-derived data. Using American Diabetes Association (ADA) definitions of greater than $126 \mathrm{mg} / \mathrm{dL}$ and $100-125 \mathrm{mg} / \mathrm{dL}$ to define T2D and prediabetes, respectively, we found that $5.8 \%$ of the self-reported healthy participants had fasting glucose measurements consistent with prediabetes, and $1.2 \%$ consistent with T2D (Fig. 2a). These results confirm prior studies showing that underdiagnosis of T2D and prediabetes is common (Table 2), similar to a previous report [32]. Analysis of the daylong CGM data revealed that many participants who self-reported as healthy exhibited postprandial glucose dysregulation (an example is shown in Fig. 2b). According to this analysis, $15 \%$ of healthy, $36 \%$ of those with prediabetes, and $81 \%$ of those with T2D 


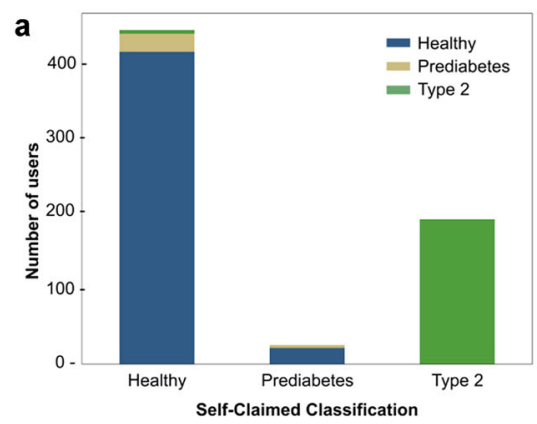

C

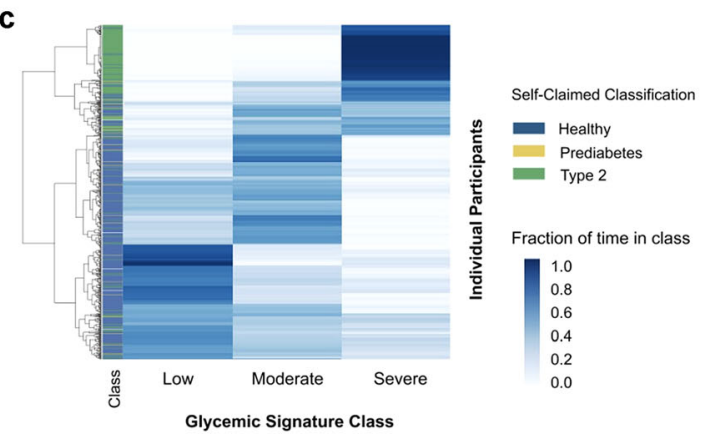

Fig. 2 Frequency of glucose dysregulation in self-claimed healthy participants. a Classification as healthy, prediabetes, and type 2 diabetes based on continuous glucose monitor values (vertical axis) in each self-claimed classification group (horizontal axis). b Variability in glucose responses to a 45-g oral glucose drink administered after an overnight fast in two healthy participants. c Heatmap showing the fraction of time individuals (shown in rows) spent in each of the glycemic signature classes, or "glucotype" (low, moderate, and high glucose values) as

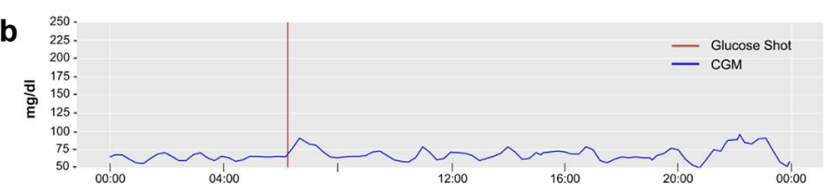

08:00 time of day HH:MM

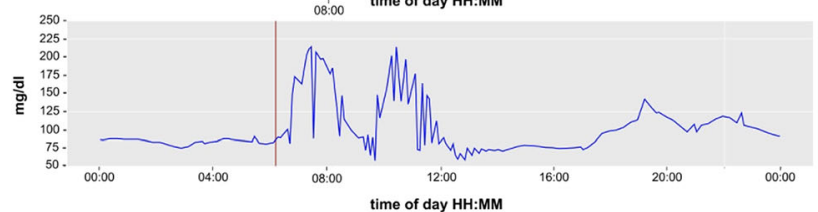

d

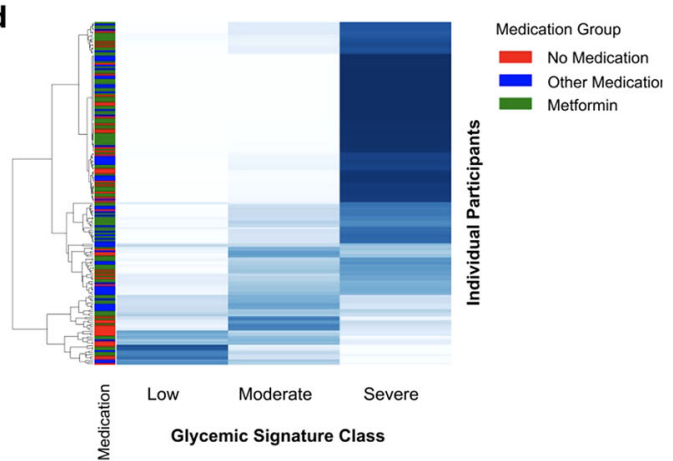

defined by Hall et al. [32]. The heat map intensity indicates the fraction of time individuals spent in each glycemic signature class with 1 being $100 \%$ of the time. Rows of individuals are arranged according to hierarchical clustering. All participants $(n=665)$ were used to create the heatmap and the left column "classification" shows the self-claimed classification group. d Heatmap showing the fraction of time T2D participants spent in each of the glycemic classes. The column on the left shows the T2Ds according to medication use

Table 2 Using January AI fasting glucose measurement users are classified as healthy, prediabetes, or T2D

\begin{tabular}{llll}
\hline Self-reported class & Healthy & Prediabetes & Type 2 diabetes \\
\hline Healthy & $92.8 \%$ & $5.8 \%$ & $1.2 \%$ \\
Prediabetes & $85.1 \%$ & $14.8 \%$ & \\
Type 2 diabetes & & & $100 \%$ \\
\hline
\end{tabular}

This table specifies the percentage of users that were reclassified using fasting glucose metrics

demonstrated CGM values of $200 \mathrm{mg} / \mathrm{dL}$ or higher at least once. Classification of individuals using the glycemic signature, or "glucotype" scale defined previously (which groups individuals on the basis of their frequency of glucose levels in low, medium, and high glycemic classes) [32], revealed $35.7 \%$ were low glucotype, $34.7 \%$ moderate, and $28.5 \%$ severe (Fig. 2c). Interestingly, $8 \%$ of individuals with self-reported T2D were in the low glucotype and some $(15 \%)$ of these were not on medication, 
indicating that their glucose was well controlled (Fig. 2d).

\section{Improvement of Time in Range over 10 Days}

We examined the effect of the CGM used with the Sugar AI app on glucose control across all study participants. On average, TIR improved significantly among the 665 participants included in this study as shown in Fig. 3a. Specifically, $51.4 \%$ demonstrated improvement in the TIR by an average of $6.4 \%(p<0.001$, Wilcoxon
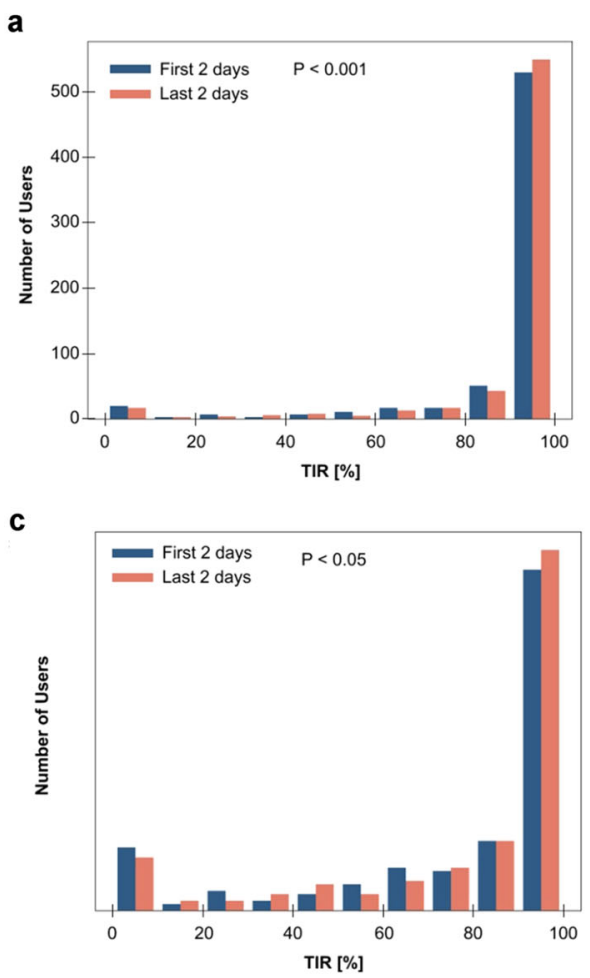

Fig. 3 Time in range (TIR) improvement across all cohorts. a Histogram of number of participants within each TIR bin over the first 2 days (days 2-3: day 1 excluded for potential CGM inaccuracy) compared to TIR over the last 2 days (days 8-9: (day 10 excluded for potential CGM inaccuracy) of the Sugar Challenge Study. TIR improved significantly overall. b An example of the first 2 days (top panel) compared to the last 2 days (bottom panel) of CGM data of a T2D participant on signed-rank test). A representative example shown in Fig. 3b. Importantly, individuals in all classes of glucose dysregulation improved their TIR significantly: those with T2D (Fig. 3c) as well as healthy nondiabetic and those with prediabetes (Fig. 3d).

\section{The Group with Poor TIR at Baseline}

In order to focus on the group that might benefit most from improvement in glucose control, we analyzed the 84 participants including those with T2D $(n=60)$ as well as those who were

b
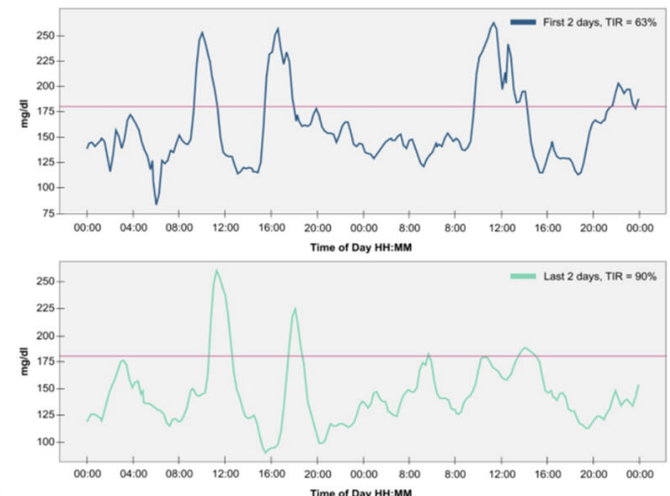

d

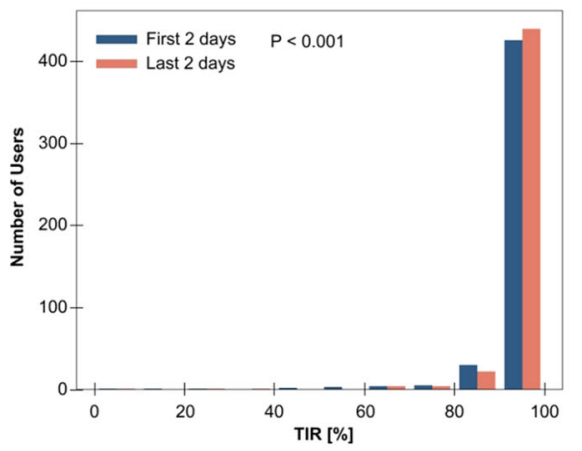

metformin. c Histogram of T2D participants within each TIR bin over days $2-3$ compared to TIR over days $8-9$ of the Sugar Challenge Study, showing significant improvement. d Histogram of healthy/prediabetes participants within each TIR bin over days 2-3 compared to TIR over days 8-9 of the Sugar Challenge Study, showing significant improvement 
healthy or self-classified as prediabetes $(n=24)$ who exhibited suboptimal TIR at the study onset, chosen as above ( less than 83\% TIR for healthy and those with prediabetes; $72 \%$ for those with T2D, corresponding to A1c diagnostic thresholds of $5.7 \%$ and $6.5 \%$, respectively, for these glycemic classifications; see "Methods" [26]). This subgroup was defined as those with "poor" TIR at baseline and was of interest as they represent a group that would potentially benefit from improved TIR.

For those with poor TIR at baseline with diabetes, 58.3\% showed TIR improvement with an average of $22.7 \%$ (Fig. $4 \mathrm{a}$ ). Among the healthy individuals and those with prediabetes whose baseline TIR was less than 83\%, 91.7\% showed TIR improvement with an average of $23.2 \%$ (Fig. 4a). Importantly, for $62.9 \%$ and $86.4 \%$ of those who improved their TIR (those with diabetes and healthy/prediabetes, respectively), the amount of improvement was greater than their own daily TIR variation, indicating this improvement was not due to daily fluctuations (Fig. 4b). For the poor TIR group, we found the TIR improved considerably from a median of $51.2 \%$ to $62.8 \%$ for all users $(p<0.004$, Mann-Whitney rank test) (Fig. 4c), $39.6 \%$ to $43.2 \%$ those with T2D (Fig. $4 \mathrm{~d}$ ), and $69.7 \%$ to $99.0 \%$ for those who are healthy/prediabetes (Fig. 4e). Figure $4 \mathrm{f}$ shows the histogram of TIR improvements for the entire group; the median improvement is $11.5 \%$.

\section{Responders vs Nonresponders}

We examined the characteristics of the individuals who improved their TIR in response to the intervention relative to those that did not. In the poor TIR group, we analyzed the characteristics of the top $25 \%$ who showed the highest TIR improvement (termed "good
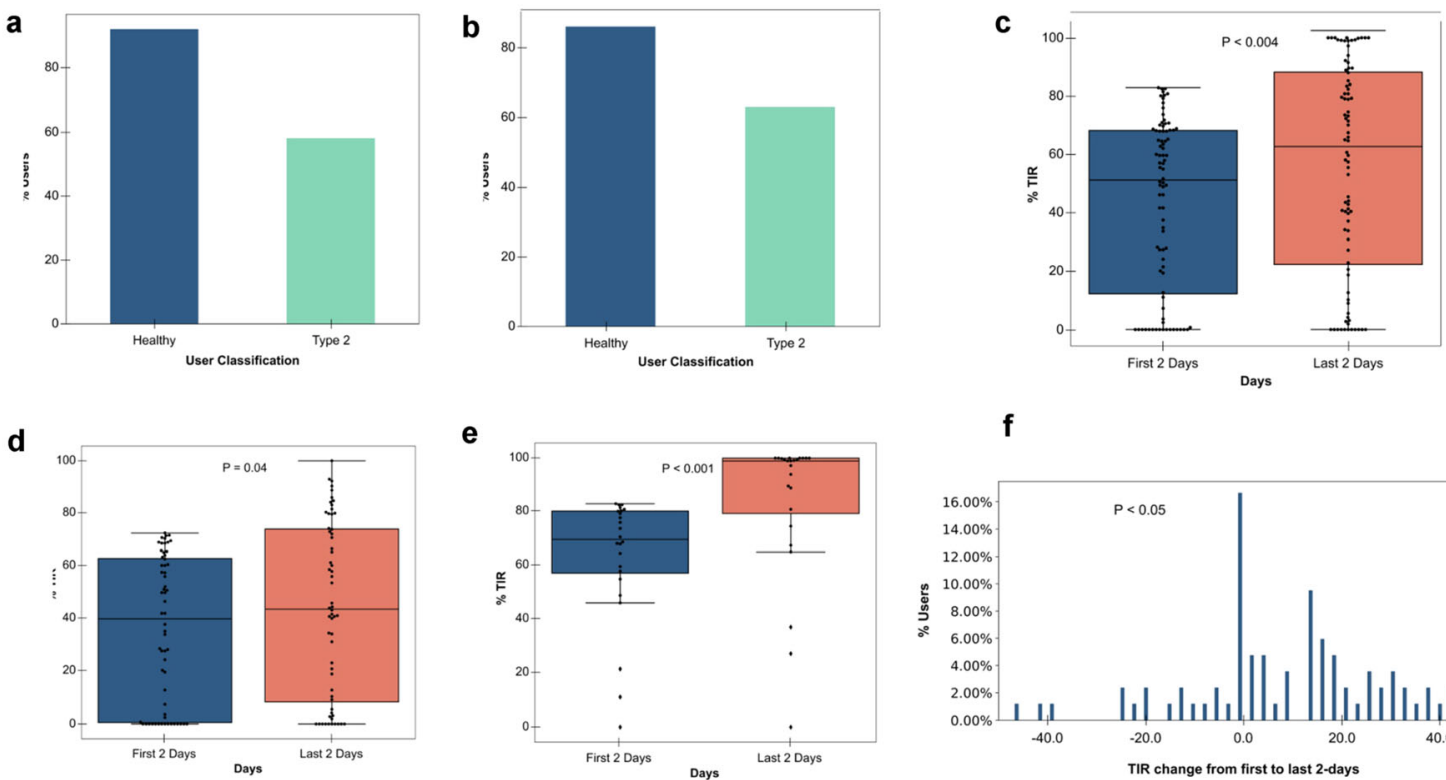

e
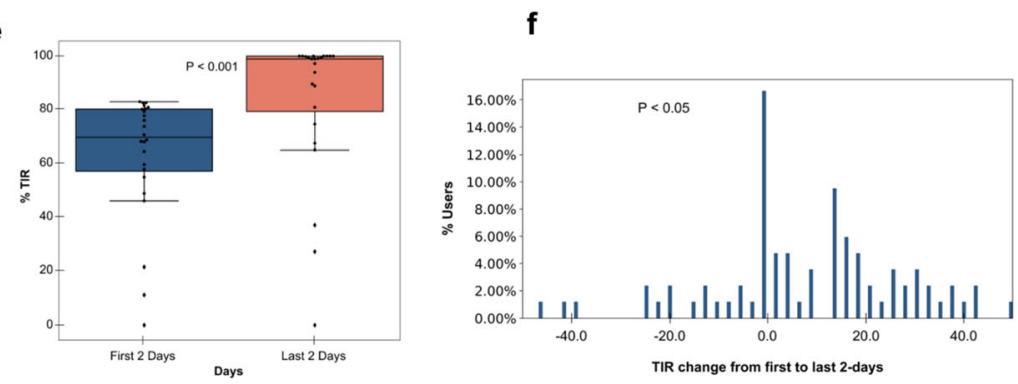

Fig. 4 The group with poor time in range (TIR) at baseline. a Percentage of users within each cohort with TIR improvement. b Percentage of users within each cohort for whom the 8-day improvement exceeded the daily TIR variation. c Comparison of days 2-3 and days 8-9 TIR for participants who started below $72 \%$ (6.5\% HbA1c equivalent for T2D users) and below $83 \%$ (5.7\% HbAlc equivalent/Healthy users) at baseline.

d Comparison of days 2-3 and days 8-9 TIR for T2D participants who started below $72 \%$ at baseline. e Comparison of days 2-3 and days 8-9 TIR for healthy/prediabetes participants who started below $83 \%$ at baseline. f Histogram of TIR improvement over T2D participants who started below $72 \%$ and healthy participants who started below $83 \%$ at baseline 
a

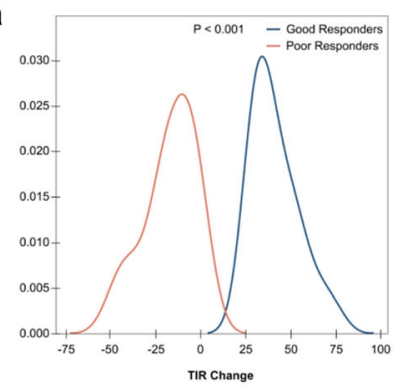

d

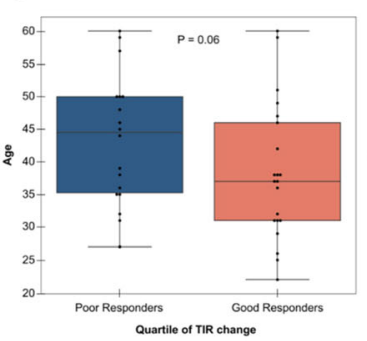

b

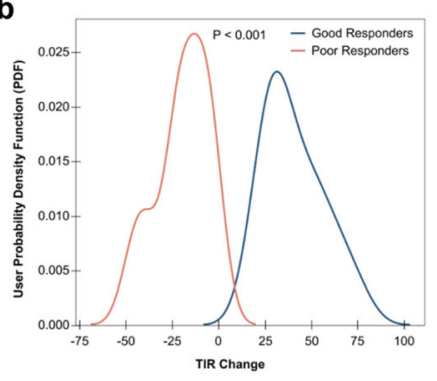

f

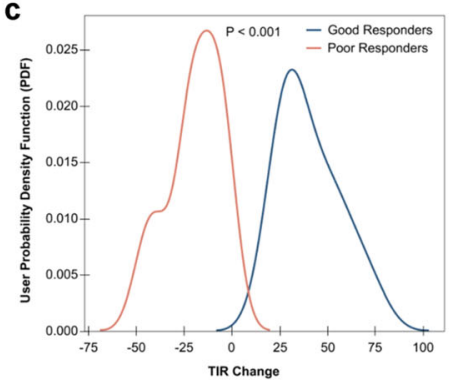

g

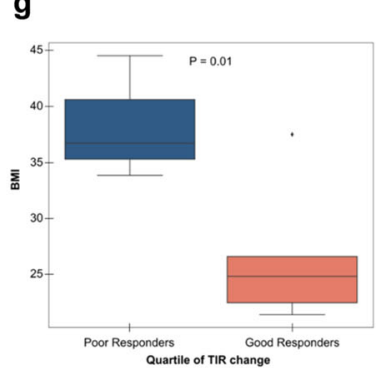

Quartile of TIR chang
Fig. 5 TIR responders vs non-responders. a All participant probability density function (PDF) of TIR change for good responders vs poor responders b T2D participant probability density function (PDF) of TIR change for good responders vs poor responders. c Healthy/prediabetes participant probability density function (PDF) of TIR change for good responders vs poor responders. d (All

responders") relative to the $25 \%$ at the bottom of this group (termed "poor responders"). The TIR improvements for these groups are shown in Fig. 5 for all (Fig. 5a), those with T2D (Fig. 5b), and those who are healthy or with prediabetes (Fig. 5c).

Analysis of these groups revealed that the good responders for all and healthy/prediabetes had a lower BMI $(p=0.05$ for all and 0.01 for healthy/prediabetes, Mann-Whitney rank test, $\mathrm{BH}$-corrected). There was a trend towards greater response in those who were younger $(p=0.06$ for all and 0.05 for healthy/prediabetes, Mann-Whitney rank test, BH-corrected). Lastly, those with classification of prediabetes or healthy nondiabetic were more likely to respond.

\section{Effect of Popular Health Bars}

We were interested in the effects of common "health" foods on TIR and therefore examined

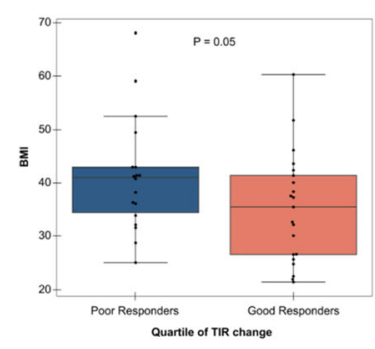

participants) Poor responders are older than good responders. e (Healthy participants) Poor responders are older than good responders. $\mathbf{f}$ (All participants) Poor responders have higher BMI than good responders. g (Healthy/ prediabetes participants) Poor responders have higher BMI than good responders

the effects of two popular nutritional bars, a Garden of Life protein bar, and a Kind bar, to determine their effects on glucose excursion. Of the 212 and 286 participants who consumed a Garden of Life protein bar and a Kind bar and adhered to our instructions in terms of fasting and logging, respectively, we found that those with normal glycemic control and the small number with prediabetes generally had excursions below $200 \mathrm{mg} / \mathrm{mL}$. By contrast, $25.9 \%$ and $19.2 \%$ of those with T2D spiked to greater than $200 \mathrm{mg} / \mathrm{mL}$ after consumption of the Garden of Life bar and Kind bar, respectively, indicating a strong hyperglycemic reaction. A substantial fraction (ca. 20\%) of participants with T2D on metformin or other antidiabetic drugs as well as T2D not on medication exhibited glucose excursions upon consuming these nutritional bars (Fig. 6). We also examined the level of glucose excursion by measuring areas under the curve (AUC) of glucose signals after bar consumption; $14.4 \%$ and $28.8 \%$ of those with T2D 


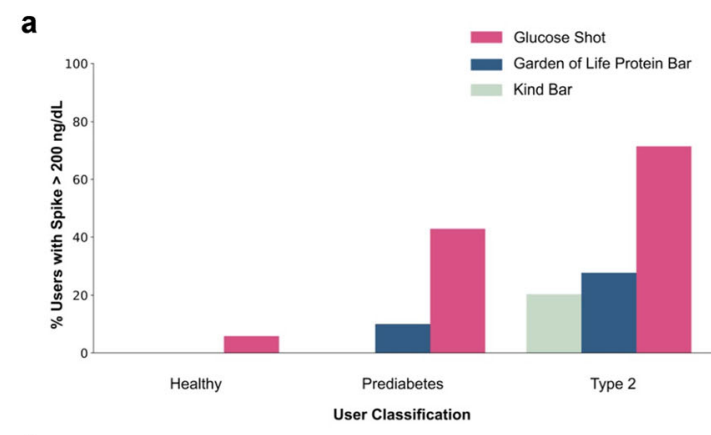

C

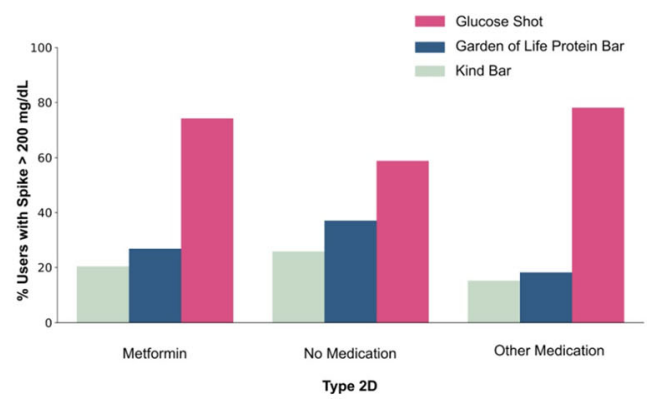

Fig. 6 Effect of standardized meals. a Percentage of participants within each cohort who spike (i.e., CGM > $200 \mathrm{mg} / \mathrm{dL}$ ) after consuming glucose shot, Kind bar, and Garden of Life high-protein Bar. b Correlation between each participant spike to Kind bar (horizontal axis) and their spike to Garden of Life bar (vertical axis) (Pearson correlation) where marker size indicates their spike to a 45-g glucose drink across healthy, prediabetes, T2D on metformin, T2D on no antidiabetic medication, and T2D on multiple medications. c Percentage of

had AUC of CGM greater than $25 \mathrm{mg} / \mathrm{dL}$ after consumption of Kind and Garden of Life bars, respectively, figures rarely $(0.6 \%$ and $0.0 \%$ respectively) observed in healthy individuals (extended data are shown in Fig. S1 in the supplementary material). Thus, these nutritional bars may not be suitable for many with T2D who want to control their glucose excursions, regardless if they are on medication.

\section{DISCUSSION}

As CGM becomes increasingly popular not just among individuals with T1D but ultimately also for those with T2D, prediabetes, and potentially even healthy individuals, it is important to understand its effects and whether its

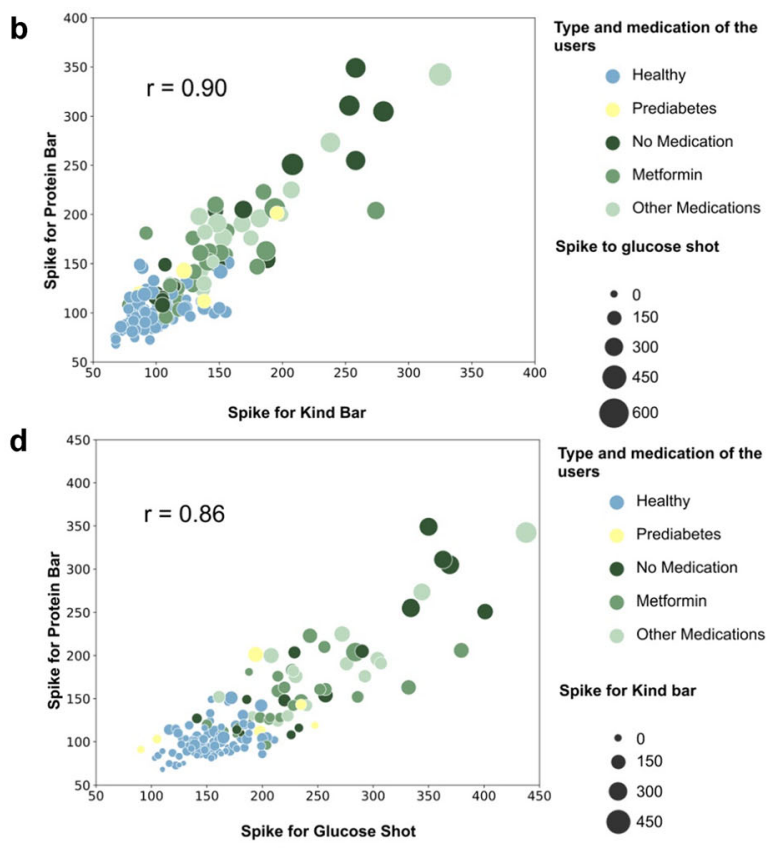

participants within each medication cohort of T2D population who spike (i.e., CGM $>200 \mathrm{mg} / \mathrm{dL}$ ) after consuming glucose drink ( $45 \mathrm{~g}$ ), Kind bar, and Garden of Life bar. $\mathbf{d}$ Correlation between each participant spike to a 45-g glucose drink (horizontal axis) and their spike to Garden of Life bar (vertical axis) (Pearson correlation) where marker size indicates their response to Kind bar across healthy, prediabetes, T2D on metformin, T2D on no antidiabetic medication, and T2D on multiple medications

concurrent use with a digital health app that links glucose patterns to modifiable behaviors, such as food choices and physical activity, is useful for the prevention and management of T2D. We have demonstrated that a 10-day use of continuous exposure to glucose levels captured by a CGM as well as logging of food, water, and physical activity tracking with data synthesis, including analysis of food consumption such as total calories, GL, and GI, and continuous insights provided by the app results in significant TIR improvement across a large and heterogeneous cohort from across the USA, spanning multiple ethnicities and both sexes, and a wide range of baseline dysglycemia. Improvement was significant in the group as a whole, and even more dramatic among those with suboptimal baseline TIR: a median of 
$11.5 \%$ for those in the poor groups (Fig. $4 a$ ) vs $0.2 \%$ for those overall. Furthermore, TIR improvement was noted not only in T2D but also in those with normoglycemia or prediabetes. In fact, those without diabetes were found to be better responders. Thus, this approach has the potential not only to improve hyperglycemia in T2D but also to delay or prevent the onset of T2D in at-risk individuals.

While one might consider that the use of this approach, like CGM, would be most useful in the most hyperglycemic patients, our results did not show this. We demonstrated that individuals who were not self-classified as having T2D were among the better responders. Other predictors of good response were young age and lower BMI. The finding that those with normoglycemia and those with prediabetes respond well to CGM plus Sugar AI and improve TIR suggests that we should not limit the use of this technology to those with T2D, and highlights that the application of CGM yields health benefits in those whose TIR is out of range after eating, and by quantifying glycemic excursions and linking them to modifiable dietary and activity behaviors, significant reductions in hyperglycemia are possible, and highlights the potential to prevent the onset of diabetes in a broader group of individuals.

In line with the above observation, the Sugar Challenge Study also revealed that when monitored by CGM, many self-reported non-diabetic participants have frequent glycemic excursions into the diabetic range: $15 \%$ of healthy and 36\% of those with prediabetes demonstrated glucose values exceeding $200 \mathrm{mg} / \mathrm{dL}$ on CGM. These results extend those of a prior study in which CGM monitoring demonstrated that $15 \%$ and $2 \%$ of self-reported "normal" or "healthy" individuals had postprandial glucose excursions to levels consistent with prediabetes and/or T2D, respectively [32]. Thus, many people have glucose dysregulation and are not aware of it. Furthermore, the present study showed that those who most frequently exhibited glucose excursions outside the upper limit of the desired range (greater than $180 \mathrm{mg} / \mathrm{dL}$ ) responded best to the CGM/Sugar AI app. These data thus highlight the value of CGM in identifying individuals with early stages of glucose dysregulation who might be missed by traditional testing methods, and who respond well to an intervention such as that presented that enables individuals to visualize glycemic excursions and link them to modifiable behaviors such as dietary intake and physical activity.

The results of this study demonstrated the value of concurrent CGM and food monitoring in evaluating the impact of foods that individuals might think are healthy. Choosing healthy foods presents a continual challenge for individuals with diabetes and even prediabetes. Foods marketed as healthy may significantly raise blood glucose levels to levels commonly observed in T2D. In the current study, a significant portion of participants (19-25\%) with T2D exhibited glucose excursions to greater than $200 \mathrm{mg} / \mathrm{dL}$ after eating popular nutritional "protein" bars. There are large numbers of these bars commercially available and many are noted for their high protein and/or fiber content. Our data indicate that these bars may not be healthy for many individuals with T2D as their sugar levels will spike into the diabetes range. CGM combined with multimodal data capture, including food logging as presented here, thus has the potential to reveal unexpected effects of foods such as health bars on glycemic responses.

Our study has several unique strengths. First, it is the largest study to date on the use of CGM for the treatment of hyperglycemia. Furthermore, there are few published studies in noninsulin-treated T2D as well as prediabetes or healthy individuals. Thus the current results are the first to demonstrate an impact on individuals in the early stages of glucose dysregulation. Our subjects were recruited from all over the USA and thus, unlike other published studies, are geographically and ethnically diverse. Lastly, this is the first study to our knowledge to evaluate the impact of CGM in concert with an application that enhances the ability of the user to interpret data relating glucose excursions to modifiable behaviors. We did not evaluate the impact of this tool with physician involvement which might produce even better results. Other limitations of the current study include the duration of 10 days. While it is impressive that significant reductions in hyperglycemia are 
noted within this short timeframe, demonstration of durability of benefit is needed. We did not engage a control group because this was an observational trial. Recruiting subjects across the country to participate in the non-intervention arm would have been exceedingly difficult in a study of this size.

Overall, the results of the current study add novel and important insights into the use of CGM-related technology to identify at-risk individuals with postprandial glucose dysregulation, and its potential to improve TIR in these individuals as well as those with T2D. Specifically, results demonstrate that a multimodal approach entailing CGM, food and activity logging, with data synthesis and continuous feedback by a smartphone app showing an overlay of glucose curves on food choices and physical activity, along with GL/GI and macronutrient tracking can improve TIR in selfdescribed healthy, prediabetic, and T2D groups. It further has the potential to enhance consumer knowledge about healthy food choices by enabling the consumer to evaluate the impact on glucose response rather than relying on the food label and advertising. In addition to improving glycemic control in patients with diagnosed T2D, this intervention is potentially important for the healthy population with the earliest postprandial glucose dysregulation and those with prediabetes since they can begin to modify their diet at an early stage prior to a diagnosis of T2D and associated symptoms (e.g., insulin resistance) and thereby likely delay the onset of T2D. Thus, the potential benefits of CGM have not been fully explored, and the capacity to gather and link data from multiple modalities may significantly enhance the clinical impact of CGM in a very large population including those at risk for diabetes.

\section{CONCLUSION}

Results from the Sugar Challenge Study indicate that short-term use of CGM along with Sugar AI app to guide lifestyle modification can be an effective approach to improve glucose regulation in healthy, prediabetes, and non-insulintreated T2D. By adjusting lifestyle choices that contribute to the development of hyperglycemia, one can potentially alleviate the risks of microvascular and macrovascular complications associated with the pathogenesis of T2D. While many of the study participants achieved glucose control rapidly, long-term studies are needed to determine if these beneficial effects are persistent.

\section{ACKNOWLEDGEMENTS}

We thank the Sugar Challenge Study participants for their dedication to advancing our understanding of approaches to improving metabolic health. We also thank Ami Bhatt for general advice and editorial comments on the manuscript.

Authorship. All named authors meet the International Committee of Medical Journal Editors (ICMJE) criteria for authorship for this article, take responsibility for the integrity of the work, and have given their approval for this version to be published.

Disclosures. Ashkan Dehghani Zahedani, Solmaz Shariat Torbaghan, Salar Rahili, Kirill Karlin, Darrin Scilley, Riya Thakkar, Maziyar Saberi, Noosheen Hashemi, Dalia Perelman, Nima Aghaeepour, Tracey McLaughlin, and Michael P. Snyder are affiliated with January, Inc.

Compliance with Ethics Guidelines. This study was performed in accordance with the Helsinki Declaration of 1964 and its later amendments. The study was approved by WCG New England IRB 120190429. Written informed consent was obtained for all participants.

Funding. This work and the Journal's rapid service fee was funded by January, Inc.

Authorship Contributions. Solmaz Shariat Torbaghan created the framework and experiment design, statistical analysis and modeling, and software development. Ashkan Dehghani Zahedani, Salar Rahili, RiyaThakkar, and Darrin Scilley helped with data analysis. Kirill Karlin, 
Maziyar Saberi, Noosheen Hashemi, Dalia Perelman helped with trial design and data collection. Nima Aghaeepour, Tracey McLaughlin, Michael Snyder helped with study design, assessment of results, and writing the manuscript.

Data Availability. The datasets generated during the current study are available upon request.

Open Access. This article is licensed under a Creative Commons Attribution-NonCommercial 4.0 International License, which permits any non-commercial use, sharing, adaptation, distribution and reproduction in any medium or format, as long as you give appropriate credit to the original author(s) and the source, provide a link to the Creative Commons licence, and indicate if changes were made. The images or other third party material in this article are included in the article's Creative Commons licence, unless indicated otherwise in a credit line to the material. If material is not included in the article's Creative Commons licence and your intended use is not permitted by statutory regulation or exceeds the permitted use, you will need to obtain permission directly from the copyright holder. To view a copy of this licence, visit http://creativecommons.org/licenses/by$\mathrm{nc} / 4.0 /$.

\section{REFERENCES}

1. National Diabetes Statistics Report, 2020, CDC. 2020. Accessed 25 Mar 2021.

2. Nathan DM, Davidson MB, DeFronzo RA, et al. Impaired fasting glucose and impaired glucose tolerance: implications for care. Diabetes Care. 2007;30(3):753-9.

3. Knowler WC, Barrett-Connor E, Fowler SE, et al. Reduction in the incidence of type 2 diabetes with lifestyle intervention or metformin. N Engl J Med. 2002;346(6):393-403.

4. Franz MJ, MacLeod J, Evert A, et al. Academy of nutrition and dietetics nutrition practice guideline for type 1 and type 2 diabetes in adults: systematic review of evidence for medical nutrition therapy effectiveness and recommendations for integration into the nutrition care process. J Acad Nutr Diet. 2017;117(10):1659-79.

5. The Diabetes Prevention Program (DPP), Diabetes Care. 2021. https://www.niddk.nih.gov/aboutniddk/research-areas/diabetes/diabetes-preventionprogram-dpp. Accessed 25 Mar 2021.

6. Ali MK, Echouffo-Tcheugui JB, Williamson DF. How effective were lifestyle interventions in real-world settings that were modeled on the diabetes prevention program? Health Aff. 2012;31(1):67-75.

7. Greenwood DA, Gee PM, Fatkin KJ, Peeples M. A systematic review of reviews evaluating technologyenabled diabetes self-management education and support. J Diabetes Sci Technol. 2017;11(5): 1015-27.

8. Kaufman N, Khurana I. Using digital health technology to prevent and treat diabetes. Diabetes Technol Ther. 2016;18(S1):S56.

9. Shan R, Sarkar S, Martin SS. Digital health technology and mobile devices for the management of diabetes mellitus: state of the art. Diabetologia. 2019;62(6):877-87.

10. Juvenile Diabetes Research Foundation Continuous Glucose Monitoring Study Group, Tamborlane WV, Beck RW, et al. Continuous glucose monitoring and intensive treatment of type 1 diabetes. $\mathrm{N}$ Engl J Med. 2008;359(14):1464-76.

11. Battelino T, Phillip M, Bratina N, Nimri R, Oskarsson $\mathrm{P}$, Bolinder J. Effect of continuous glucose monitoring on hypoglycemia in type 1 diabetes. Diabetes Care. 2011;34(4):795-800.

12. Juvenile Diabetes Research Foundation Continuous Glucose Monitoring Study Group, Bode B, Beck $\mathrm{RW}$, et al. Sustained benefit of continuous glucose monitoring on $\mathrm{A} 1 \mathrm{C}$, glucose profiles, and hypoglycemia in adults with type 1 diabetes. Diabetes Care. 2009;32(11):2047-9.

13. Beck RW, Riddlesworth TD, Ruedy K, et al. Continuous glucose monitoring versus usual care in patients with type 2 diabetes receiving multiple daily insulin injections. Ann Intern Med. 2017;167(6):365-74.

14. Ehrhardt NM, Chellappa M, Walker MS, Fonda SJ, Vigersky RA. The effect of real-time continuous glucose monitoring on glycemic control in patients with type 2 diabetes mellitus. J Diabetes Sci Technol. 2011;5(3):668-75.

15. Vigersky RA, Fonda SJ, Chellappa M, Walker MS, Ehrhardt NM. Short- and long-term effects of realtime continuous glucose monitoring in patients 
with type 2 diabetes. Diabetes Care. 2012;35(1): 32-8.

16. Haak T, Hanaire H, Ajjan R, Hermanns N, Riveline $\mathrm{J}-\mathrm{P}$, Rayman $\mathrm{G}$. Flash glucose-sensing technology as a replacement for blood glucose monitoring for the management of insulin-treated type 2 diabetes: a multicenter, open-label randomized controlled trial. Diabetes Ther. 2017;8(1):55-73.

17. Ajjan RA, Abougila K, Bellary S, et al. Sensor and software use for the glycaemic management of insulin-treated type 1 and type 2 diabetes patients. Diabetes Vasc Dis Res. 2016;13(3):211-9.

18. Petrie JR, Peters AL, Bergenstal RM, Holl RW, Fleming GA, Heinemann L. Improving the clinical value and utility of CGM systems: issues and recommendations: a joint statement of the European Association for the Study of Diabetes and the American Diabetes Association Diabetes Technology Working Group. Diabetologia. 2017;60(12): 2319-28.

19. Allen NA, Fain JA, Braun B, Chipkin SR. Continuous glucose monitoring counseling improves physical activity behaviors of individuals with type 2 diabetes: a randomized clinical trial. Diabetes Res Clin Pract. 2008;80(3):371-9.

20. Cox DJ, Taylor AG, Moncrief M, et al. Continuous glucose monitoring in the self-management of type 2 diabetes: a paradigm shift. Diabetes Care. 2016;39(5):e71-3.

21. Yoo HJ, An HG, Park SY, et al. Use of a real time continuous glucose monitoring system as a motivational device for poorly controlled type 2 diabetes. Diabetes Res Clin Pract. 2008;82(1):73-9.

22. Wada E, Onoue T, Kobayashi T, et al. Flash glucose monitoring helps achieve better glycemic control than conventional self-monitoring of blood glucose in non-insulin-treated type 2 diabetes: a randomized controlled trial. BMJ Open Diabetes Res Care. 2020;8(1):e001115.
23. Beck RW, Bergenstal RM, Riddlesworth TD, et al. Validation of time in range as an outcome measure for diabetes clinical trials. Diabetes Care. 2019;42(3):400-5.

24. American Diabetes Association. 6. Glycemic targets: standards of medical care in diabetes-2021. Diabetes Care. 2021;44(Supplement 1):S73-84.

25. Fabris C, Heinemann L, Beck R, Cobelli C, Kovatchev B. Estimation of hemoglobin A1c from continuous glucose monitoring data in individuals with type 1 diabetes: is time in range all we need? Diabetes Technol Ther. 2020;22(7):501-8.

26. Vigersky RA, McMahon C. The relationship of hemoglobin A1C to time-in-range in patients with diabetes. Diabetes Technol Ther. 2019;21(2):81-5.

27. Scheff SW. Chapter 8: nonparametric statistics. In: Scheff SW, editor. Fundamental statistical principles for the neurobiologist. London: Academic; 2016. p. 157-82.

28. Marino MJ. Chapter 3: statistical analysis in preclinical biomedical research. In: Williams M, Curtis MJ, Mullane K, editors. Research in the biomedical sciences. London: Academic; 2018. p. 107-44.

29. McIntosh AM, Sharpe M, Lawrie SM. Research methods, statistics and evidence-based practice. In: Johnstone EC, Owens DC, Lawrie SM, McIntosh AM, Sharpe M, editors. Companion to psychiatric studies. 8th ed. St Louis: Churchill Livingstone; 2010. p. 157-98.

30. Nichols K. Chapter 20 false discovery rate procedures. In: Friston K, Ashburner J, Kiebel S, Nichols $\mathrm{T}$, Penny $\mathrm{W}$, editors. Statistical parametric mapping. London: Academic; 2007. p. 246-52.

31. Noble WS. How does multiple testing correction work? Nat Biotechnol. 2009;27(12):1135-7.

32. Hall H, Perelman D, Breschi A, et al. Glucotypes reveal new patterns of glucose dysregulation. PLoS Biol. 2018;16(7):e2005143. 